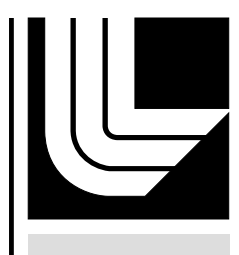

LAW RENCE LIVERMORE N A T IO N A L LABORATORY

\title{
UCRL-TR-231805
}

\section{Program Manager's Questions for LL03-LL254DP-PD03}

M. Heffner

June 15, 2007 
This document was prepared as an account of work sponsored by an agency of the United States Government. Neither the United States Government nor the University of California nor any of their employees, makes any warranty, express or implied, or assumes any legal liability or responsibility for the accuracy, completeness, or usefulness of any information, apparatus, product, or process disclosed, or represents that its use would not infringe privately owned rights. Reference herein to any specific commercial product, process, or service by trade name, trademark, manufacturer, or otherwise, does not necessarily constitute or imply its endorsement, recommendation, or favoring by the United States Government or the University of California. The views and opinions of authors expressed herein do not necessarily state or reflect those of the United States Government or the University of California, and shall not be used for advertising or product endorsement purposes.

This work was performed under the auspices of the U.S. Department of Energy by University of California, Lawrence Livermore National Laboratory under Contract W-7405-Eng-48 


\section{Program Manager's Questions \\ for}

LL03-LL254DP-PD03

(Directional Fast-Neutron Detectors for Identification/Location of Fissile Material)

Scientific/Technical Soundness

1. What are the current project goals and are they well defined?

The goals are described in the "Experimental Plan" talk on slides 3,8,10 and 11. The main goals are:

- What is the true pointing accuracy in the field?

- What is the effect of various background and scattering sources?

- What is the realistic sensitivity of the TPC approach?

- What is the best fieldable configuration?

The specific tasks to answer these questions are described in the series of talks presented in this review.

\section{Please compare demonstrated results and proposed / envisioned system with comparable commercial systems and other systems under development, if any.}

This is addressed on slide 4 of the Introduction talk. There is only one other method to detect the direction of the fast neutrons, the scatter camera. It is difficult at this early stage to pick which technology is better, but we think that the TPC has distinct advantages over the scatter camera.

- No limit to efficiency in principle

- $4 \pi$ acceptance

- full tracking (not just 2 points)

- specific ionization along track - particle identification

- resolution scales with linearly with electronics channel count (scales with the square for a scatter camera)

3. Is the technical program plan reasonable and likely to achieve the project objectives?

The program plan can be summarized as:

- Build the prototype hardware

- Test a few candidate gases

- Evaluate the performance with a ${ }^{252} \mathrm{Cf}$ source

- Design the fieldable TPC with the knowledge gained from this work 
There have been some concerns over the $\mathrm{H}_{2}$ at high pressure. Given this feedback the plan has been expanded slightly to include alkanes to lower the pressure and removed the need for $\mathrm{H}_{2}$.

4. Please describe technical progress to date and indicate how well it meets the agreed to milestone and deliverable schedules.

The TPC has been built and the first demonstration of neutron direction measurement has been made in ${ }^{4} \mathrm{He}$. All milestones and deliverables have been met. The rate of progress has been simply a function of the money available.

5. A number of gasses ranging from $\mathrm{H} \& \mathrm{He}$ to the alkanes are mentioned as potential detection media in the experimental unit. Please briefly discuss the advantages and disadvantages of each with respect to detection performance visa vis (a) detection efficiency, (b) pointing accuracy, (c) minimum number of interactions to locate a source, (d) safety, (e) ease of use, ...

There are 2 basic categories of gas choices, Hydrogen and $3 \mathrm{He}$. The Hydrogen can be further broken down into $\mathrm{H}_{2}$ gas and the alkanes. The $\mathrm{H}_{2}$ gas and the alkanes should perform approximately the same for a,b,c and e. There are questions about the safety of $\mathrm{H}_{2}$ in the minds of the users, and the alkanes are an alternative that have lower flammability and lower pressure for the same efficiency.

The ${ }^{3} \mathrm{He}$ reaction is a fully constrained measurement of the neutron angle so it provides more information per scatter, but has a lower cross section so there are less scatters. One of the experimental tasks is to look at the pointing accuracy of these two cases.

6. Some potential end users have expressed concern over several fielability issues like; size and weight, complexity of operation, high pressure gas volume, microphonics resulting from wire or strip electrodes, gas handling concerns (explosive, corrosive, volatile, ...). How can these risks be mitigated or eliminated. Can these issues be discussed in a way that places the risk in perspective to other operational risks that may be more familiar?

Size and weight

The nominal size that we have considered is $1 \mathrm{~m}^{3}$. The actual size for an application depends on the efficiency required for detection. The weight of a $1 \mathrm{~m}^{3}$ TPC should be less than $100 \mathrm{lb}$ which can be easily be carried by 2 people. If careful engineering is applied to this problem the weight could be significantly less than 100lb.

complexity of operation 
The operation could be made very simple. There would be two plugs on the TPC vessel: one power cord, and an Ethernet cable to a laptop. The user turns on the laptop and runs a program that points to the source, or provides an image of the neutron source and energy spectrum.

high pressure gas volume

The use of alkanes will reduce the pressure requirement. An example is a $1 \mathrm{~m}^{3}$ propane TPC at $22.5 \mathrm{psi}$ will have $20 \%$ efficiency.

microphonics resulting from wire or strip electrodes

In a fieldable design we could make use of gain structures that are well supported and therefore very stiff. In addition, the resonant frequency could be adjusted to put any oscillation out of the way and perhaps if need a notch filter could be used.

gas handling concerns

The thought at this point is that it would be a sealed as system so there is no gas handling for the user. The system may need some periodic maintenance by the manufacturer to refresh the gas. Depending on how good the vessel is constructed, refresh cycles could hopefully be years. NID could also help extend the refresh cycle by lowering the sensitive of the TPC to air contaminates.

In the "Experimental Plan" talk, slides $6 \& 7$ put the use of these gases in perspective with common hazards.

\section{Please explain the NID effect in more detail and discuss impacts on performance.}

NID is caused by introducing an electronegative gas such as $\mathrm{CS}_{2}$ that collects the ionization making the $\mathrm{CS}_{2}$ molecule negative. The $\mathrm{CS}_{2}$ then drifts in the electric field instead of the electron. This lowers the drift speed, the diffusion and the electron attachment. The reason to use the gas is to allow for large volumes where the diffusion and electron attachment can become a problem.

8. Background is mentioned as a confounding issue when ${ }^{4} \mathrm{He}$ is used as a detection medium. It is described in your LCP that this is due to contamination by alpha emitters though the source is never mentioned. One is lead to assume that this results from residual gas species in the counting gas reservoir. It would appear that this could be eliminated or minimized by gas purification prior to filling. If indeed $4 \mathrm{He}$ is chosen as the desired fill gas, what steps are envisioned to mitigate the background issue? 
${ }^{4} \mathrm{He}$ is not an optimal gas for the TPC. It is 4 times more massive than a $\mathrm{H}$ and this difference reduces the angular resolution of the detector. We used ${ }^{4} \mathrm{He}$ as a as that we could get up and running quickly. If a user decided that the lower resolution is acceptable the gas could be cleaned up and low activity material selected to reduce the alpha contamination. We did not specify an alpha source in the LCP because we did not spend the time to investigate the source of the alphas.

9. One of the major tasks in this project is to increase the number of readout channels. Can you please briefly describe how the channel count and distribution around the chamber affects detection efficiency and pointing resolution? What is considered the ultimate resolution?

The channel density is determined by the length of a track in the gas; for good tracking at least 10-20 points along the track. The total number of channels is the density times the area of the readout plane. With fewer channels one gives up on either good tracking by lowering the density, or instrumenting the full volume. The first lowers the pointing resolution and particle identification, the second lowers the efficiency of the detector.

The ultimate resolution is more difficult to quantify because it depends on the environment. From the TPC standpoint it could be quite good. Sub-degree accuracy could probably be accomplished, but the scattering of the neutrons getting to the detector would probably be a much larger factor.

Management/Execution

1. Who are the primary individuals working on this project and what are their roles? Please include your subcontractors as they are instrumental to the success of this project.

Mike Heffner - Current PI for the project.

Leslie Rosenberg - Currently professor at University of Washington. Previous $\mathrm{PI}$ for this project. Currently in charge of the DAQ development.

Celeste Winant - Postdoc working on the preamps.

Norm Madden - Electrical engineer supporting the low noise analog signal extraction and post processing electronics associated with this project.

Adam Bernstein - interact with sponsor regarding project status and funding recruit/identify project staff (Rosenberg/Heffner/Winant/Madden) - assist in defining project goals and deliverables - about $5 \%$ effort.

Darrell Carter - Mechanical Technician

Michael Hotz - UW graduate student. DAQ development.

2. Please provide the background of these individuals relevant to their roles. 
Mike Heffner has worked on TPCs for the last 14 years. The main TPC projects have been, the EOS TPC, STAR TPC, Fission TPC, and has worked on the nTPC for all but the first year.

Leslie Rosenberg has long experience with particle detection. He designed the nTPC and was the PI for the project until the end of FY06. He is a Fellow of the American Physical Society, with citation his challenging measurements.

Adam Bernstein has worked on radiation detector development for nonproliferation for eight years. He has designed and deployed ton scale scintillator detectors for monitoring plutonium content in nuclear reactors, and for active scanning of cargo containers. He is a member of the XENON collaboration, which uses a dual phase xenon TPC to search for dark matter. $\mathrm{He}$ is the LLNL Subject Matter Expert and point of contact for the SNM Detection portfolio in NA-22, the NNSA sponsor of the nTPC project.

Norm Madden supported the early demonstration phase of this TPC until his recent retirement this past June. He is currently a consultant to this project and has 34 years of experience in nuclear instrumentation.

Celeste Winant has worked on TPCs at LLNL for low energy radiation detection for 3 years. She received her PhD in physics from UC Berkeley in 2003 having researched the temperature anisotropy of the cosmic microwave background with the balloon-borne far-infrared bolometric receiver, MAXIMA. She has over ten years of hands-on experience in detector physics, with expertise in cryogenics and analog readout electronics development.

3. Is there any potential for additional university or small business collaboration for any unclassified components of this project (potentially funded though our university grant or SBIR programs)?

Certainly in the later stages of this project when a fieldable design is built, a partnership with a company would be highly desirable. We currently have university involvement so we could consider funding them thought a direct grant.

4. Are there any classification issues that need attention with respect to the tools, techniques, methods, or capabilities being developed under this project?

None that we are aware of.

5. Is this project team engaged in similar work sponsored by DNDO, DTRA, or other NNSA offices? If so, please describe technical area and 
application area deconflict between the NA-22 work and that for these other organizations.

No

6. Is this project team engaged in similar work sponsored by other WFO or IWFO? If so, please describe how the technical work is complementary and integrates into this NA-22 sponsored effort. Also, please describe the applications of interest at the appropriate classification level.

No

7. Please upload and properly account for reports or publications generated by this project in webPMIS.

We will do that.

8. Do you know of other NA-22 projects would receive benefits from successful completion of this project? Or are there other NA-22 projects that could benefit your effort?

Our work on low cost and small sized Ethernet-based data acquisition with 100-200 channel capability may benefit gamma ray imaging, neutron scatter camera, multi-channel readouts such as the UltraSpec superconducting calorimeter. Of course other users are also working along these lines.

9. Who are competitors for developments of this or similar technology in the labs, universities, and industry and how are you distinguishing yourselves from them?

We are fairly certain that we are the most advanced group working on light gas TPCs for these applications, and we believe our technological progress is more advanced than the competing scatter camera work funded by NA-22.

SNL is working on a neutron scatter camera. Presentations available to us indicate proof of principle with a two pixel (1 front 1 rear) system. Our device already exceeds this channel count readout and has true tracking capability with multiple hits per track (8 currently, 192 shortly depending on funding) Based on discussions with BNL personnel, BNL has explored light gas TPCs. As far as we know this work is unfunded. We have heard from our sponsor that DTRA is funding for a ${ }^{3} \mathrm{He}$ based TPC development project. We are uncertain of the status of this project. 
10. Following the normal cycle program build for NA-22, it was decided that there was sufficient potential end user support to warrant continuation of this project. Since the decision was out of cycle, only limited resources were applied $(\$ 200 \mathrm{k}$ of the original $\sim \$ 500 \mathrm{k}$ proposed). The current CR (continuing resolution) of the DOE budget has eliminated the availability of additional resources to apply against this project until such time that the CR is lifted. It is possible, perhaps likely, that the DOE will be operating under CR for the duration of FY07. Please describe the impact of this fiscal reality on your project execution and planning for next year. Revision to your LCP will be required to either extend project duration or to reduce the deliverables set. I look forward to learning the recommendations of the review committee.

With only $40 \%$ of the funds we can not complete the same tasks that we could with $\$ 500 \mathrm{k}$. The project has become accustom to breaks in the funding and although this introduces inefficiency into the project we can plan around this issue. The main impact is that the project will be delayed in reaching its goals.

Potential User Impact

1. What user agencies would be expected to be interested in the capabilities of the proposed systems? What contacts have been made and have they shown an interest or made suggestions? What fora or venues have you considered for presenting the results of this work to the non-proliferation and national security communities? Have you planned for IC or other non-proliferation community representation during any briefings or demonstrations?

DHS, DOE and DoD and DoS all have jurisdiction in areas of interest for this technology. DOE end users include emergency response teams, which have expressed interest in the technology, and possibly others. DHS DNDO (the nearterm research arm of DHS) has expressed interest in neutron directionality and has issued explicit calls for this class of technology. We would expect DoS and DOE interest for treaty monitoring activities such as verification of operations at Pu and HEU production facilities, as part of the IAEA safeguards or other nonproliferation and fissile material control agreements.

We have proposed to our program sponsor to give briefings to several end users in DC mediated by NA-22 personnel. We have presented the technology at NA22's ProTech conference in FY06. We intend to give a briefing to interested NP community representatives as soon as we have a high channel count system in operation.

2. Non-proliferation applications in search and ship-effect neutron discrimination have been discussed in the non-proliferation community within NNSA. Please provide a brief description of how you would 
envision a fieldable TCP addressing these applications, discussing the potential advantages over other approaches.

The ship effect refers to an increase in neutron backgrounds in neutron counters, observed on ships, possibly due to hadronic and muonic interactions with the ship. A neutron TPC might in principle allow discrimination against these backgrounds through directionality or energy information, but further characterization of the effect and the detector performance is needed to determine any advantage. Generally, we believe that for search applications in warehouses, parking lots, or for that matter ships, the ability to point with few degree to few tens of degrees accuracy, and have high confidence that the observed data are fission energy neutrons, will be decisive advantages relative to other methods, including 'proximity imaging' with $\mathrm{He} 3$ detectors, spectroscopy without directionality, or the equivalent methods using gamma ray detectors. Confirmation of this claim requires detailed analysis of concepts of operation, and probably field trials, which are in our year 3 work plan but which we have not yet pursued. The main disadvantage is that the absolute detection efficiency can be greatly reduced by hydrogenous shielding, and directionality is compromised by either high or low-Z shielding.

\section{A pointing accuracy of 10's of degrees from a field based TPC is projected based on previous performance. Can you discuss the efficacy of this spatial performance for search applications?}

In the context of search, even 180 degree pointing can provide an important advantage, for example identifying the lane in which a car with SNM is traveling past an nTPC equipped portal. Further it is important to note that 10's of degrees accuracy assumes 5 sigma detection above a Poisson background at 40 meters in air with just ten events - more dwell time will improve the pointing accuracy.

The TPC pointing accuracy is not limited in principle to 10's of degrees. This is the performance with a weak signal ( $\sim 10$ neutrons) and the hydrogen containing gases. The intrinsic resolution of the TPC could be sub-degree, but all directional neutron detectors (including the scatter camera) have the same problem of neutron scatters before reaching the detector. This will most likely be the limiting factor to pointing resolution.

\section{Utility of the TPC in active interrogation scenarios is also alluded to in your LCP. Can you briefly discuss an envisioned approach and the advantage over other detection approaches?}

Since the TPC is a $4 \pi$ detector with pointing one could imagine an active scenario where an interrogation source is not collimated, flooding the target and the return fast neutrons would be localized by the pointing of the TPC. A more concrete example would be a van with the TPC and source could drive up to a target (say a Truck) and ping it to see if there is SNM in the truck. In this way the detector is mobile and the target fixed. This is the opposite of the standard 
scanning of the target though a portal. This speculative idea has not been developed as an approach and therefore was not discussed in detail in the LCP. 